Historic, Archive Document

Do not assume content reflects current scientific knowledge, policies, or practices. 



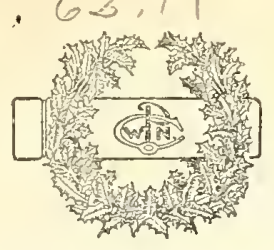

East Washington at Sixth St.

\section{Dortlared, Oregore}

\section{FRUT TREE SEEDLINGS \& OTHER LINING OUT STOCK}

We book all Orders with the understanding that same shall be Void should injury befall the stock from Flood, Drought, Fire, Frost or other catses Befond iegntrol.

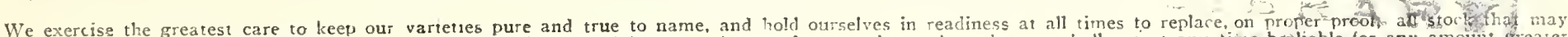

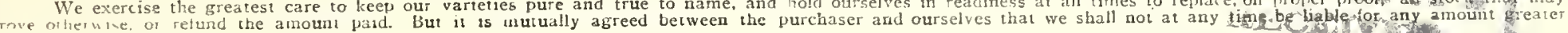
than the original price.

TERHS OF PAYMENT:

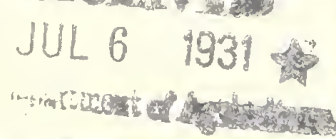

TO THE TRADEDNAY

1. CASH PIAN- If you send us cash with your order you may deduct $3 \%$ from the amount and we will pack it free or charge.

2. PART PAYHENT- If you will send us one third of the amount with your order we will forward it C. O. D. for balance.

3. CHARGE ACCOUNT- On these our terms are sjzty days to customers who have estaolished thejr credit vith us. New charge accounts will be opened only on receipt of satisfactory references with tine for due investigation.

Stock from beds or field will be puddled and well packed in moss, and that from pots careiully wrapped so as to reach you in first class condition.

We re;ommend that orders for Iining out stock go forward by express and in the absence of definite instructions they will be shipped in thet manner.

On oraers for one thousand or more of one $\nabla$ rietjety you may deduct one cent each from the list price provided it is $\$ 8.00$ or more per hunared, or if less than $\$ 8.00$ deduct one half cent.

PIEASE NOTE. In this list "C" indicates stock is grom from cuttings and "S" from seed. "O" indicates never transplanted, and each "x" one transplanting.

\section{CONIFERS}

Price

Arborvitae, Am. Golden-Thuja oc. arrea

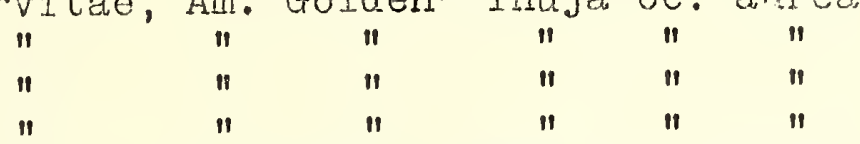

Am. Globe-Thuja oc. globosa

Times

transp. Size

"

$"$

$n$

$"$

\section{Golaen Berimans-I.}

Go $n$

Cedar, Atlas-Cedrus atlantica

" Deodar-Cedrus deodara

" Lebanon-Cedrus libani

Cryptomeria, PIune- C. japonica elegans " " Cypress, Italian- C.semoervirens fastigiata

$\begin{array}{cllll}\text { " " } & \text { " } & \text { " } & \text { " } & \end{array}$

C
C
C
C
C
C
C
C

$\mathrm{S}$

S

$\mathrm{S}$

C

C $\mathrm{x}$

S

S $x x$

$\mathrm{xx}$

$\mathrm{x} x$

$x . x$

$x$

$x$

$\mathrm{x}$

$x$

$x$

0

$x$

$x$

$\mathrm{x}$

$\mathrm{X}$

$x$

$12-15 "$

$10-12 "$

8-10"

$6-8 "$

$3-4 "$

$6-8 "$

$4-6 "$

$2-411$

$6-8 "$

$4-6 "$

$6-8 "$

$6-8 "$

$4-6 "$

$10-121$

$8-10^{\prime \prime}$

$6-8^{\prime \prime}$
From Per 100

Fjela $\$$ \$5.00

" 12.00

" 10.00

" 8.00

2"Pots 8.00

Field 15.00

$" 12.00$

" 8.00

Beds 12.00

" 10.00

$1 \quad 12.00$

3 "Iots 12.00

3" " 10.00

Field 15.00

" 12.00

" 10.00 

CONIFERS: Continued.

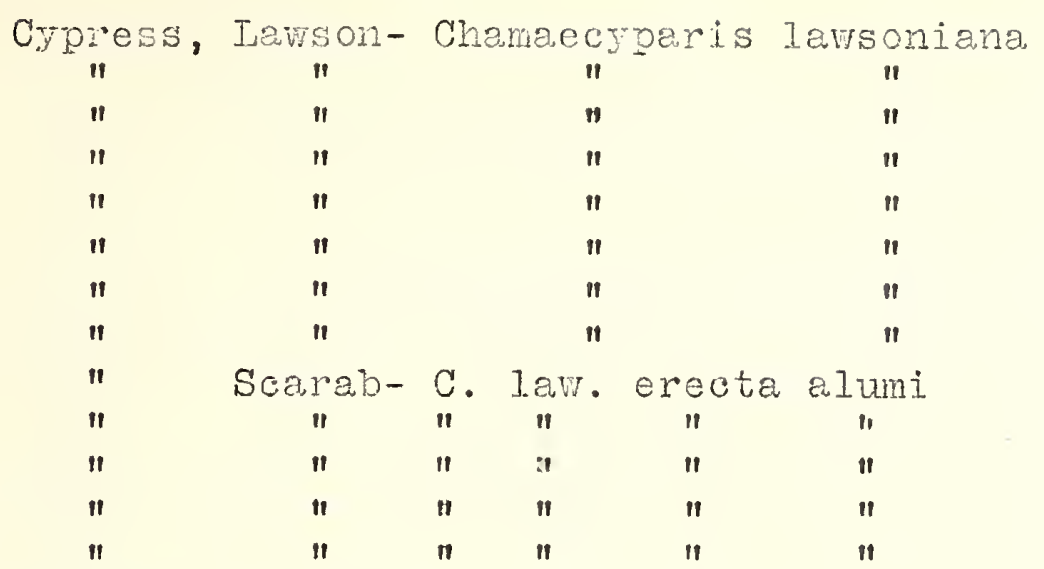

Fir, Thite-Abies concolor

Hemlock, Canada- I'suga canadensis

Juniper, Chinese- Juniperus chinensis

$\begin{array}{lccc}\text { " } & \text { Irish- Juniperus hibernica } \\ " 1 " & \text { " } & \text { " } & \text { " } \\ \text { " } & \text { " } & \text { " } & " 1 \\ \text { " } & \text { " } & \text { " } & \text { " }\end{array}$

Pine, Mugho- Pinus montana mughus " Old Mexico- Pinus montezumae

Retinospora, Heath- R. ericoides

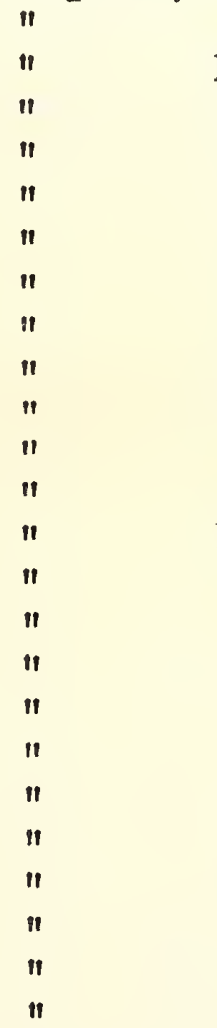

Moss- R. squarrosa veitchi

\begin{tabular}{|c|c|c|c|}
\hline " & $n$ & " & $n$ \\
\hline$" 1$ & $n$ & $n$ & "1 \\
\hline$\|$ & $n$ & $n$ & "1 \\
\hline 11 & $" 1$ & 11 & 11 \\
\hline$n$ & $n$ & 11 & " \\
\hline 11 & $\|$ & $\|$ & $n$ \\
\hline 11 & n & $n$ & $n$ \\
\hline
\end{tabular}

Plume-Cham. pisifera plumosa.

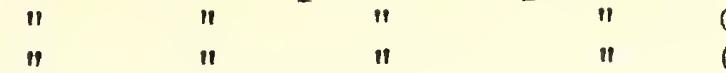

Plume, Golden- C. p. aurea.

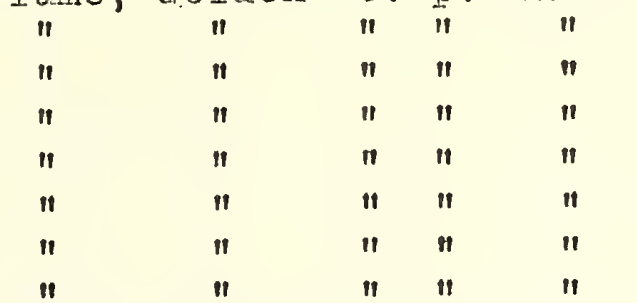

Threadbranch- R. Pilipera
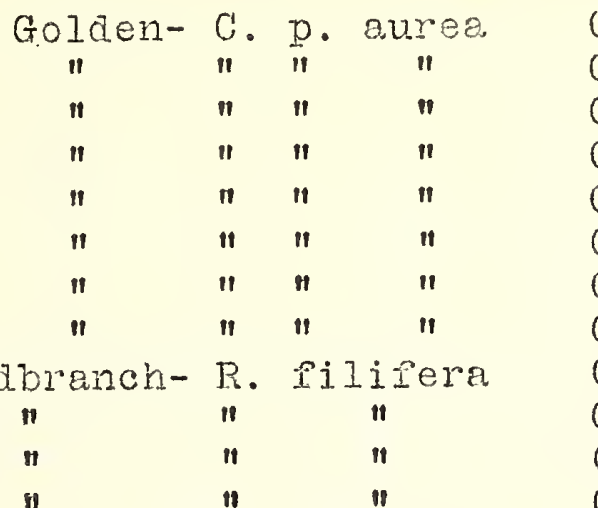

C
Times

transp. Size

$C$
$C$
S
S
S
S
S
S
C
$C$
$C$
$C$
$C$
C
S

$\mathrm{xx}$

$x x$

$Y$

$x$

$x$

X.

X.

X

X

$x$
$x$

$x$

$x$

$x$

C $x$

S 0

$\mathrm{S}$

C

C

c

¿

C

C

C

$\mathrm{S}$

S

C

C

C

C

C

C $x$

C $x$

C $x$

C $x$

C $x$

C

C

C

C $\mathrm{xx}$

$\begin{array}{ll}C & x \\ C & x\end{array}$

C $x$

C $x$

C $X$

C $x$

$\begin{array}{ll}\text { C } & x \\ \text { C } & x\end{array}$
Size
$1.0-12 "$
$8-10 "$

15-18"

$12-15 "$

$10-12 "$

$8-10 "$

$6-8 "$

$4-6^{\prime \prime}$

$12-15 "$

$10-12 "$

$8-10^{\prime \prime}$

$6-8$ "

$4-6 "$

$1-2 "$

㮃"

4-6"

$2-4 "$

$12-15 "$

$10-12 "$

$8-10 "$

$6-8 "$

$10-1.21$

3-10"

$6-8 "$

$1-2 "$

$2-4 "$

$4-6 "$

$2-4 n$

$10-12 "$

$8-10 "$

$6-8 "$

$10-12 "$

$8-10 "$

$6-8 "$

$4-6 n$

$2-4^{\prime \prime}$

$10-12^{n}$

8-10"

$6-8^{\prime \prime}$

$10-12 "$

8-10"

$6-8^{\prime \prime}$

$4-6 " 1$

$8-10^{\prime \prime}$

$6-8^{\prime \prime}$

$4-6^{\prime \prime}$

$2-4^{\prime \prime}$

10-12"

$8-10 "$

6-8"

$4-6$ "
Price

From Per 100

Field 15.00

12.00

18.00

15.00

12.00

10.00

8.00

6.00

18.00

].5.00

12.00

10.00

8.00

Beds

3.00

2.00

Hield 8.00

5.00

18.00

12.00

10.00

8.00

10.00

8.00

6.00

5.00

Beds 5.00

Field $\quad 8.00$

5.00

20.00

18.00

15.00

15.00

12.00

10.00

8.00

5.00

20.00

18.00

1.5 .00

20.00

18.00

15.00

12.00

12.00

10.00

8.00

5.00

15.00

12.00

10.00

8.00

In this list "C" indicates stock grom from cuttings and "s" from seed. "O" indicoses never transplanted, and each "x" one transplanting. 

CONIFIFS: Continued.

Times

transp. Size

$\underset{\|}{\text { Spruce, Norwat - Picea excelsa }}$

$\mathrm{S}$

"

"

Colorndo Blue-Picer pu

$" 1 "$

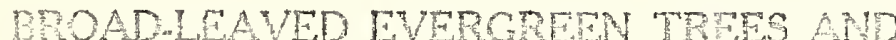

Aucubr, Goldanst- Aucuba jap. Variegate

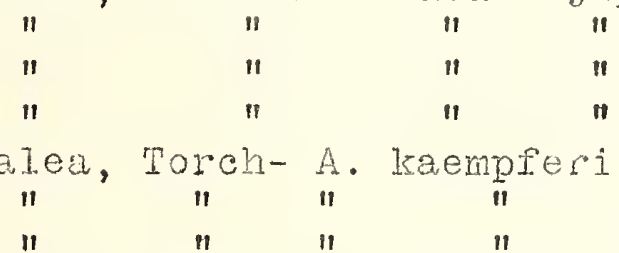

Barberry, Darwin- Berberis darwini

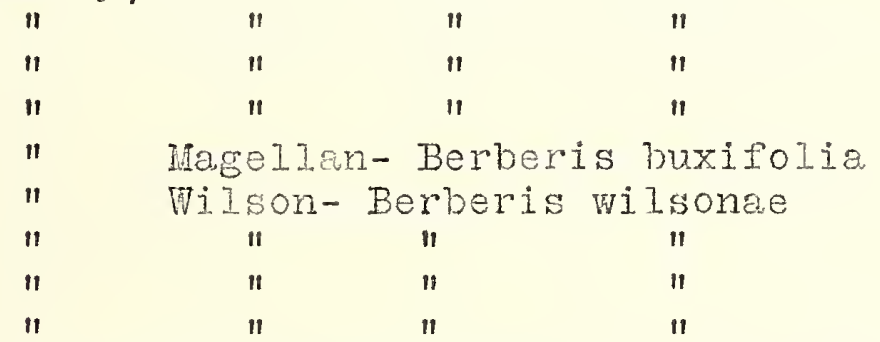

Box, Truetree- Buxus semperv. arborescens

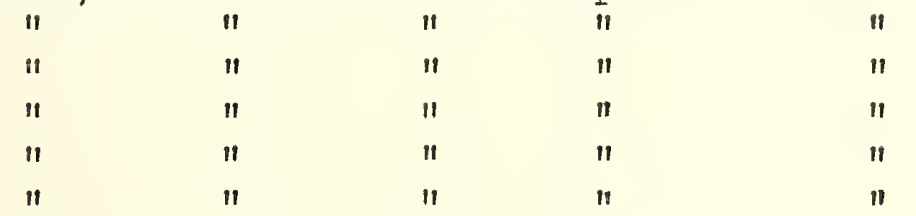

Votoneaster Acutifolia

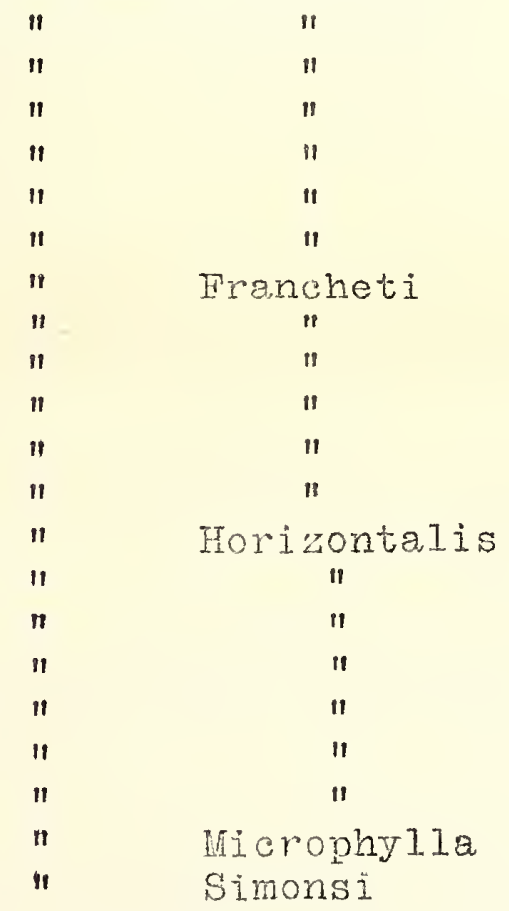

(⿸丆口

$x$

$x$
$x$
$x$
$x$
$x$
$x$
$x$
$x$
$x$
$x$
$x$
$x$
0
0
0

$\mathrm{XX}$

XX

$\mathrm{Xx}$

$x$

$x$

$x$

$x$

$x$

$x$

$x$

$\mathrm{X}$

$x$

$x$

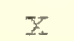

$x$

$x$

$x$

$x$

$x$

$x$

$x$

$x$

is

$x$

$x$

$\mathrm{C} \quad \mathrm{X}$

S $x$

C I

c

C

$6-811$

$6-8 "$

$4-6 "$

$2-41$

$15-18^{\prime \prime}$

$12-1511$

$10-12^{\prime \prime}$

$8-10^{\prime \prime}$

$6-8 n$

$4-6 n$

$2-411$

$15-18^{\prime \prime}$

$12-15 "$

$10-121$

$8-10^{\prime \prime}$

$6-8^{\prime \prime}$

$4-6^{\prime \prime}$

$12-15 "$

$10-12 "$

$6-8 "$

$4-6 "$

$2-411$

$2-4 n$

$6-8 "$
$2-41$

$1-2 "$

$\frac{1}{3}-2 " 1$
$\frac{3}{2}-1 "$
$\frac{3}{2}-I^{\prime \prime}$
Price

Exom Per 100

Field क्w?.00

1. 00

3.00

2.00

Bejs

SHPUDS

\begin{tabular}{|c|c|c|}
\hline $3-10^{\prime \prime}$ & Eield & $\$ 15.00$ \\
\hline $6-8^{\prime \prime}$ & " & 12.00 \\
\hline $4-611$ & 11 & 10.00 \\
\hline $2-4 n$ & $" 1$ & 8.00 \\
\hline $4-611$ & 11 & 12.00 \\
\hline $2-411$ & $"$ & 10.00 \\
\hline $1-211$ & 11 & 7.00 \\
\hline $3-10^{n}$ & 11 & 12.00 \\
\hline $6-8^{\prime \prime}$ & $"$ & 10.00 \\
\hline $4-611$ & 11 & 8.00 \\
\hline $2-411$ & "1 & 5.00 \\
\hline $6-8^{\prime \prime}$ & $"$ & 30.00 \\
\hline $10-7.211$ & $"$ & 15.00 \\
\hline $8-10^{\prime \prime}$ & Beds & 6.00 \\
\hline $6-8^{\prime \prime}$ & if & 5.0 \\
\hline $4-6^{\prime \prime}$ & $"$ & 4.0 \\
\hline
\end{tabular}

10-12" Fiela 12.00

$8-10 " \quad " \quad 10.00$

$8-10^{\prime \prime}$

$10-12^{\prime \prime}$

10.00
8.00

6.00

4.00

3.00

15.00

12.00

10.00

8.00

6.00

4.00

2.00

15.00

12.00

10.00

8.00

6.00

4.00

12.00

10.00

8.00

6.00

4. 00

2.00

4.00

6.00

10.00

In this list "C" indicates stock grown from cuttings and "S" from seed. "o" indicates never transplanted, and each "z" one trensplanting. 


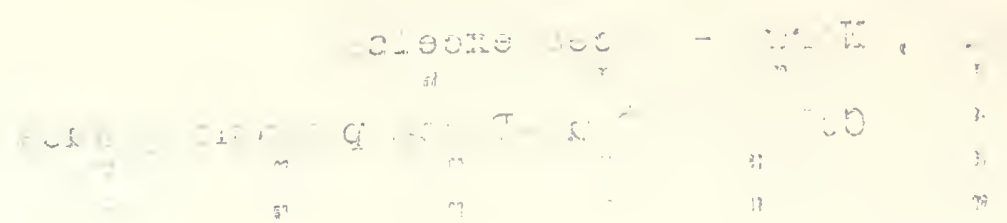

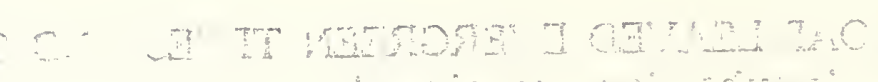

1

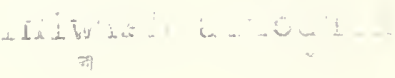

C

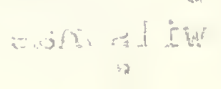

asc. i 
BROAD IEAVTED IEVERGREIESS: Continued.

Dephine, Winter- D. odora

Esco,Ilonia rubra

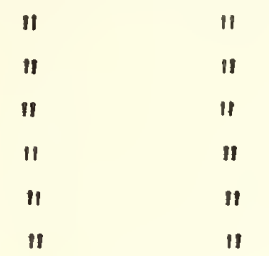

Zuonyms, Tvergreen- E. japonicus " japonicus aureus

Heath, Biscay- Elica, mediterranea

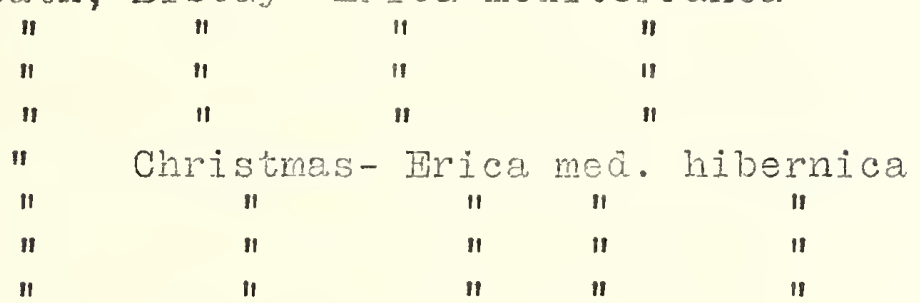

Honeysuckle- Ionicera nitida

$$
\text { " " " }
$$

Iaurel, English- Laurocerasus officinalis

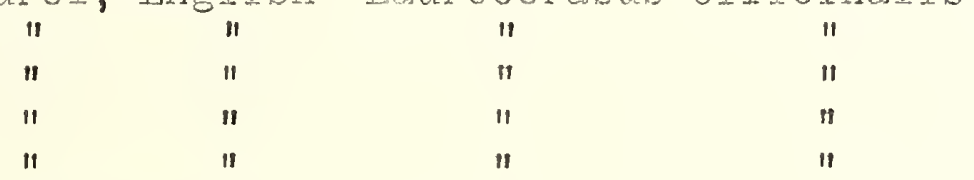

Oregon Grape- Mahonia aquipolia

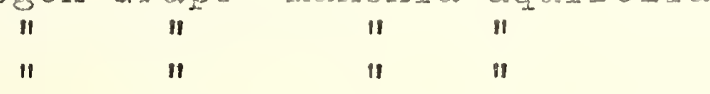

Privet, Golden-Jigustrum ovalj.e. aureum

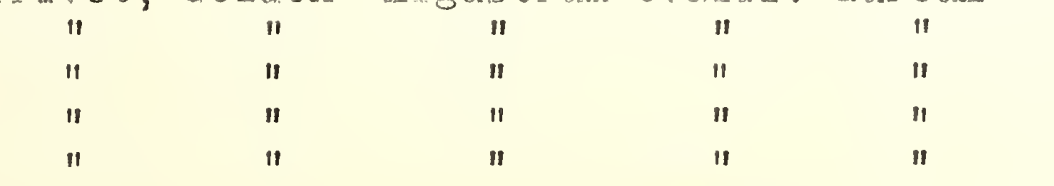

\section{DECIDUOUS SIRESBS}

Times

transp. Size

c

C $x$

C X

C $X$

C $x$

C $x$

C I

C $X$

C $x x$

C

C

C

C

c

C

C$$
\text { c }
$$

o$$
x
$$$$
X
$$$$
X
$$$$
\text { X }
$$$$
x
$$$$
x
$$$$
x
$$$$
x
$$

$$
\begin{aligned}
& X \\
& X \\
& X \\
& X \\
& X \\
& X \\
& X \\
& X \\
& X \\
& X \\
& X \\
& X \\
& X \\
& X \\
& X \\
& X \\
& X \\
& X \\
& X \\
& X \\
& X \\
& X \\
& X \\
& X \\
& X \\
& X \\
& X \\
& X \\
& X \\
& X \\
& X \\
& X \\
& X \\
& X \\
& X \\
& X \\
& X \\
& X \\
& X \\
& X
\end{aligned}
$$$$
x
$$$$
\text { z }
$$

$3-4 "$

18-24"

$15-18 "$

$12-15^{\prime \prime}$

$1 \mathrm{C}-12^{\prime \prime}$

$8-10^{\prime \prime}$

$$
6-81
$$

$$
\text { 4-6" }
$$

$$
8-10 "
$$

$10-1211$

$$
6-8 "
$$

$8-10^{\prime \prime}$

$6-81$

$4-6 "$

$2-4 "$

$8-70^{\prime \prime}$

$6-8 "$

$4-6 n$

$2-411$

$10-12 "$

$8-10^{\prime \prime}$

$$
6-8 "
$$

$15-18 n$

$12-15^{\prime \prime}$

] $0-12 "$

$$
8-10^{\prime \prime}
$$$$
\text { 6-8" }
$$

]. $\mathrm{C}-1.2$ "

8-10"

$6-8:$

$15-18^{\prime \prime}$

$12-15^{\prime \prime}$

$10-12^{\prime \prime}$

8-1.0"

$6-8 n$
Price

Erom Per I00

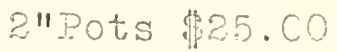

Field

18.00

15.00

12.00

10.00

8.00

6.00

4.00

10.00

8.00

5.00

1.0 .00

8.00

6.00

4.00

10.00

8.C0

6.00

4.00

10.00

8.00

6.00

18.00

15.00

12.00

10.00

8.00

10.00

8.00

6.00

15.00

12.00

10.00

8.00

6.00

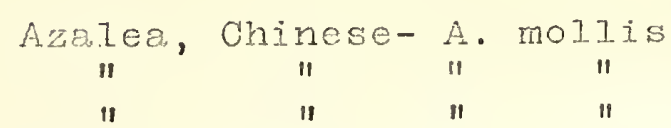

Kerria- K. japonica

Spirea Thunberg-S. thumbergi

$\begin{array}{lccc}" ~ & " 1 & " 1 \\ " & " 1 & " \\ " & \text { Venhoutte- S. Vanhouttei }\end{array}$

Heigela, Pink- h. rosea

remarix, Acican- T. airicena

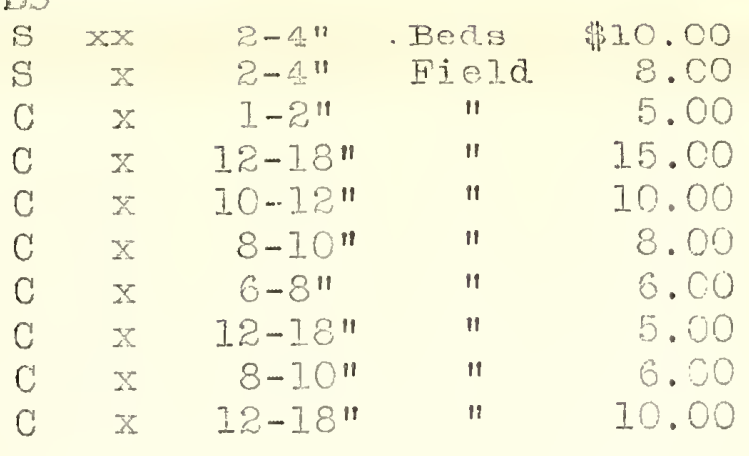

\section{VINES}

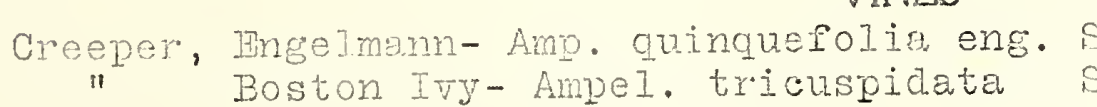

In this 3ist "c" indicates stock grown erom cuttings and "S" from bes. "oi indicates never trangplanted, and each "rl one transplanting". 



\section{FRUIT AND SHADE TREE SEEDLINGS}

\section{CLEAN COAST GROWN}

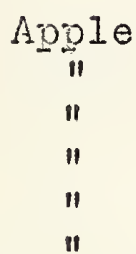

Grade

Pear, Calleryana, French, Japan and Ussuriensis

$1 / 4^{\prime \prime} \quad$ branched $1 / 4 "$

$\# 1,3 / 16^{\prime \prime} \&$ up

湆1, 3/16" \& up

\#2, 2/16" to $3 / 16 "$

\#3, about $2 / 16 "$ straight

branched.

straight
$1 / 4 " 1$

\#I, 3/16" \& up

\#2, 2/16" to 3/16"

\#3, about 2/16"

$1 / 4 n$

Cherry, Mazzard

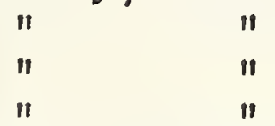

Cherry, Bahaleb

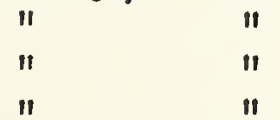

Plum, Myrobolan

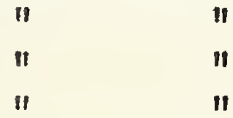

$\underset{\text { "Peach, Lovell seedings }}{\text { " }}$

Persimmon, De Lotus seedlings

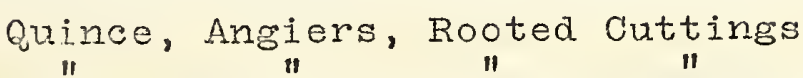

$\# 1,3 / 16 "$ \& up

\#2, 2/16" to 3/16"

\#3, 8bout $2 / 16^{\prime \prime}$

$1 / 4 "$

\#1, 3/16" \& up

"it2, 2/16" to $3 / 16 "$

"t:3, about $2 / 16 "$

$1 / 4=$

\#1, 3/16" \& up

\#2, 2/16" to $3 / 16 "$

\#3, about $2 / 16 "$

HI, 3/16" \& up

\#2, $2 / 16^{\prime \prime}$ to $3 / 16$ "

"ti, $3 / 16^{\prime \prime}$ \& $u p$

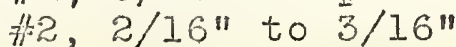

\#1, $3 / 1$ 6" \& u.p

"1/2, $2 / 1.6 "$ to $3 / 16 "$
Per 1000

$\$ 15.00$

13.00

12.00

10.00

8.00

4.00

20.00

16.00

12.00

10.00

20.00

16.00

12.00

8.00

16.00

12.00

8.00

6.00

16.00

12.00

8.00

6.00

10.00

8.00

20.00

15.00

20.00

15.00

55.00

45.00

35.00

25.00

20.00

25.00

20.00

125.00

100.00

75.00

60.00

50.00

Correspondence invited on quantity orders. 
